\title{
Congruency sequence effects and cognitive control
}

\author{
Tobias Egner \\ Northwestern University, Chicago, Illinois
}

\begin{abstract}
Congruency effects in selective attention tasks are subject to sequential modulation: They are smaller following an incongruent stimulus than following a congruent one. This congruency sequence effect has been interpreted as reflecting conflict-driven adjustments in cognitive control (conflict adaptation) or, alternatively, episodic memory effects of stimulus-response association (feature integration). The present article critically reviews support for these rival accounts in the experimental literature and discusses the implications thereof for assessing behavioral and neural signatures of cognitive control processes. It is argued that both conflict adaptation and feature integration contribute to the congruency sequence effect but that their respective contributions can be isolated experimentally. Studies that have pursued this isolation strategy have gained important insights into cognitive control processes. Finally, other factors, such as expectancies, may also contribute to the congruency sequence effect, and thus their potential role needs to be carefully examined and, if found significant, integrated into current models of cognitive control.
\end{abstract}

In recent years, medial and lateral prefrontal cortices have been hypothesized to play a crucial role in cognitive control, the flexible regulation of behavior in the pursuit of internal goals (Botvinick, Braver, Barch, Carter, \& Cohen, 2001; Miller \& Cohen, 2001; Posner \& DiGirolamo, 1998; Ridderinkhof, Ullsperger, Crone, \& Nieuwenhuis, 2004). Cognitive control is essential in situations in which routine behavior does not produce satisfactory performance - for example, when prepotent response tendencies have to be overcome. Therefore, performance on congruency tasks, such as the Stroop task (Stroop, 1935), has long been considered a useful model for probing controlled processing (Cohen, Dunbar, \& McClelland, 1990; Cohen, ServanSchreiber, \& McClelland, 1992). In particular, it has been argued that first-order congruency sequence effects - that is, when the effect is found to be smaller following an incongruent stimulus than following a congruent oneprovide a direct window onto online adjustments in cognitive control (Botvinick et al., 2001). In turn, neuroimaging studies have harnessed the congruency sequence effect to tease apart brain regions putatively involved in different aspects of cognitive control operations (Botvinick, Nystrom, Fissell, Carter, \& Cohen, 1999; Egner, Delano, \& Hirsch, 2007; Egner \& Hirsch, 2005a, 2005b; Etkin, Egner, Peraza, Kandel, \& Hirsch, 2006; Kerns, 2006; Kerns et al., 2004; Stürmer \& Leuthold, 2003; Stürmer, Leuthold, Soetens, Schröter, \& Sommer, 2002; for a review, see Botvinick, Cohen, \& Carter, 2004).

However, the congruency sequence effect is susceptible to alternative explanations that do not require the contribution of cognitive control mechanisms (Hommel,
Proctor, \& Vu, 2004; Mayr, Awh, \& Laurey, 2003). These alternative explanations have introduced considerable uncertainty into the interpretation of this effect and its associated neuroimaging data and have triggered a fastgrowing experimental literature aimed at clarifying the psychological determinants of congruency sequence effects, which has yielded mixed conclusions (Burle, Allain, Vidal, \& Hasbroucq, 2005; Nieuwenhuis et al., 2006; Notebaert, Gevers, Verbruggen, \& Liefooghe, 2006; Ullsperger, Bylsma, \& Botvinick, 2005; Verbruggen, Notebaert, Liefooghe, \& Vandierendonck, 2006; Wendt, Kluwe, \& Peters, 2006; Wühr, 2005; Wühr \& Ansorge, 2005). The present article provides a critical evaluation of the implications of this literature for assessing behavioral and neural signatures of cognitive control processes. First, different types of congruency effects, the congruency sequence effect, and the two main theoretical accounts thereof-namely, conflict adaptation and feature integration - are introduced. Second, the behavioral and neurophysiological literature is examined for support of these models. Third, a possible alternative account of the congruency sequence effect, based on expectancies, is highlighted.

\section{Congruency Effects in Flanker, Stroop, and Simon Tasks}

The body of empirical findings on the congruency sequence effect comprises a blend of data collected with versions of the Eriksen flanker task (Eriksen \& Eriksen, 1974), the Stroop task (MacLeod, 1991; Stroop, 1935), and the Simon task (Simon, 1969). Commonalities and

T. Egner, t-egner@northwestern.edu 
differences in the way in which congruency effects arise among these tasks will be briefly delineated here within the framework of Kornblum's dimensional overlap model (Kornblum, Hasbroucq, \& Osman, 1990; Kornblum \& Lee, 1995; H. Zhang, Zhang, \& Kornblum, 1999). Within a stimulus-response (S-R) forced choice task, stimuli and responses can have various dimensions (or features), each of which is either relevant or irrelevant with respect to task requirements. Congruency effects can arise whenever dimensions overlap - that is, when different dimensions of stimuli or responses (or both) display a high degree of perceptual, conceptual, or structural similarity. When overlapping stimulus and/or response dimensions are incongruent with each other, they give rise to conflicting representations and/or response tendencies; these need to be disambiguated in order for the correct response to be selected, resulting in slowed response times (RTs) in incongruent versus congruent conditions. Congruency effects can stem from overlap between relevant and irrelevant stimulus dimensions ( $\mathrm{S}-\mathrm{S}$ overlap), overlap between the relevant stimulus dimension and a response dimension (relevant S-R overlap), and overlap between an irrelevant stimulus dimension and a response dimension (irrelevant S-R overlap).

In a typical flanker paradigm, stimuli consist of strings of letters, partitioned into a central letter (the task-relevant dimension) and two (or more) flanking letters (the taskirrelevant dimension), and subjects are required to indicate the identity of the central letter by pushing response buttons that are arbitrarily mapped to different letters. Typically, each dimension has only two possible values. For example, stimulus values may consist of the letters $\mathrm{S}$ and $\mathrm{H}$. The flanker task contains an S-S overlap, since task-relevant and task-irrelevant stimulus dimensions are drawn from the same set of letters. Accordingly, the flanker letters can be congruent (SSS, HHH) or incongruent (SHS, HSH) with the central letter, and subjects respond more slowly to incongruent than to congruent stimuli (Eriksen \& Eriksen, 1974). This congruency effect can be further broken down into two additive subcomponents (Eriksen \& Eriksen, 1974). First, an incongruent S-S overlap is associated with conflicting stimulus representations, resulting in stimulus conflict. In addition, since the two stimulus values are usually mapped onto different responses, an incongruent flanker stimulus is also associated with response competition, resulting in response conflict. The typical flanker task entails neither relevant nor irrelevant S-R overlaps, since the response dimension (an arbitrarily mapped buttonpress) does not overlap with either of the stimulus dimensions and thus cannot be mapped congruently or incongruently to those dimensions ${ }^{1}$ - that is, performance would not be affected if the allocation of response buttons were reversed between $\mathrm{S}$ and $\mathrm{H}$.

In the Stroop task, stimuli comprise words for colors (e.g., RED) that are printed in colored ink; they thus entail a word meaning dimension and an ink color dimension, and subjects are required to identify the ink color. Word meaning and ink color are conceptually related, representing an S-S overlap, and can therefore be either congruent or incongruent with each other (i.e., the word RED could be printed in red ink or in green ink), and incongruent stimuli are identified more slowly and less accurately than congruent ones (MacLeod, 1991; Stroop, 1935). In most current applications of the Stroop task, particularly in neuroimaging studies, each ink color is arbitrarily mapped to a different response button, rendering Stroop congruency effects to be a function solely of $\mathrm{S}-\mathrm{S}$ overlap, ${ }^{2}$ as in the flanker task. Also as in the flanker task, an incongruent Stroop stimulus is associated with both stimulus conflict and response conflict if different stimulus values are mapped onto different manual responses (De Houwer, 2003; Milham et al., 2001; van Veen \& Carter, 2005; H. Zhang \& Kornblum, 1998). It should be noted, however, that the locus (and nature) of stimulus conflict is arguably different in these two tasks, since the S-S overlap in the Stroop task occurs at a semantic level, whereas in the flanker task, it may occur at an earlier, perceptual level (see, e.g., Nieuwenhuis et al., 2006; van Veen \& Carter, 2005).

In a typical Simon task, stimuli comprise a generic shape (e.g., a rectangle) in one of two colors (e.g., red or green) that is presented either to the left or to the right of a central fixation. Subjects are required to categorize the color of the stimulus by pushing response buttons with their left (e.g., for green) or right (e.g., for red) hand (Lu $\&$ Proctor, 1995; Simon, 1969). Simon stimuli are twodimensional in that they are defined by color and spatial position, but they do not entail any S-S overlap, since color and space are unrelated - that is, they cannot be congruent or incongruent with each other. The Simon task also does not entail relevant S-R overlap, since the color values are arbitrarily mapped onto left or right manual responses. The Simon task does, however, contain an irrelevant S-R overlap: The irrelevant stimulus dimension (spatial location: left vs. right) overlaps with the relevant response dimension (manual response: left vs. right). Therefore, the irrelevant stimulus dimension can be either congruent (e.g., a green stimulus presented on the left side) or incongruent (e.g., a red stimulus presented on the left side) with the response dimension, and incongruent stimuli are associated with substantially slower performance ( $\mathrm{Lu} \&$ Proctor, 1995; Simon, 1969).

\section{Congruency Sequence Effects}

First-order sequential modulation of congruency effects was originally reported by Gratton, Coles, and Donchin (1992) in the context of a letter flanker task. In addition to the expected main effect of congruency, the authors observed an interaction between previous and current trial congruency, in which congruency effects were smaller following incongruent stimulus trials than following congruent ones. As the schematic depiction of this effect on RT in Figure 1A shows, congruent stimuli following a congruent stimulus (CC trials) are associated with fast responses, and incongruent stimuli following a congruent stimulus (CI trials) are associated with slow responses, resulting in a large current trial congruency effect following a congruent stimulus. On the other hand, RT to incongruent and congruent stimuli that follow an incongruent stimulus (II and IC trials, respectively) falls between these extremes, resulting in a smaller congruency effect when the previous stimulus was incongruent. This 

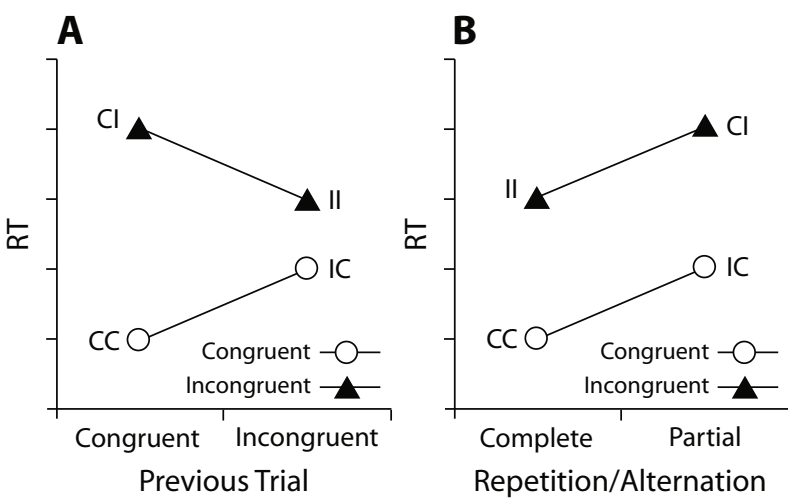

Figure 1. Hypothetical response time (RT) data schematically depict the congruency sequence effect. (A) The data are plotted as a function of previous and current trial congruency $(\mathrm{CC}$, previous congruent/current congruent; $C I$, previous congruent/current incongruent; IC, previous incongruent/current congruent; II, previous incongruent/current incongruent). (B) The same data are replotted as a function of current trial congruency and of whether all (complete) or only some (partial) stimulus and response features repeat or alternate from the previous to the current trial.

reduced congruency effect stems from both a decrease in RT to incongruent stimuli (i.e., II trials are faster than CI trials) and an increase in RT to congruent stimuli (i.e., IC trials are slower than CC trials). This effect is not related to a speed-accuracy trade-off, since accuracy rates typically display the inverse pattern of results.

\section{The Conflict-Monitoring Account}

The conflict-monitoring model holds that the congruency sequence effect stems from conflict-driven adjustments in cognitive control (Botvinick et al., 2001). An incongruent trial leads to the simultaneous activation of competing responses, or response conflict. This conflict is detected by a dedicated conflict-monitoring mechanism, thought to reside in the anterior cingulate cortex (ACC), which triggers an up-regulation in cognitive control, thought to be implemented by the lateral prefrontal cortex (LPFC), in order to overcome the conflict. Therefore, the level of cognitive control is high during trials that follow an incongruent trial. In contrast, congruent trials are not associated with response conflict and do not result in a temporary up-regulation of cognitive control. Hence, the level of control is low during trials that follow a congruent trial. An increase in cognitive control is thought to result in stronger attentional biasing of information processing in line with current task demands, such that the processing of task-relevant information is enhanced relative to the processing of task-irrelevant information. This reduces the influence of the irrelevant stimulus dimension on performance, resulting in a decreased congruency effect following incongruent stimulus trials than following congruent ones. Therefore, the faster RTs for II trials than for CI trials are the result of reduced interference from incongruent distractors, whereas the slower RTs for IC trials than for CC trials are the result of reduced facilitation from congruent distractors (see Figure 1A). This interpretation of the congruency sequence effect has been dubbed conflict adaptation (Mayr et al., 2003).

\section{The Feature Integration Account}

The feature integration account, advanced by Hommel et al. (2004), is based on episodic memory effects of S-R associations and does not invoke cognitive control mechanisms. Independently of this theory, Mayr et al. (2003) have expressed concern that repetition priming effects may mediate the congruency sequence effect. However, since repetition priming constitutes a special case of feature integration effects, it will not be treated separately here. According to the feature integration model, if a given stimulus and response co-occur in time, then stimulus and response features are integrated into a common episodic memory representation (an event file). A subsequent activation of any one of these features automatically coactivates the other features. A complete S-R repetition, therefore, results in particularly fast responses, since the repeated coactivation of these features takes little effort (this situation represents the repetition priming effect; see, e.g., Pashler $\&$ Baylis, 1991). Moreover, a complete alternation of all stimulus and response features is also thought to be processed relatively swiftly, since no previous feature binding has to be overcome. On the other hand, if a stimulus feature is repeated but the response is required to change (or vice versa), processing will be slowed down, since the previous feature binding has to be overcome. In other words, complete repetition and complete alternation of all stimulus and response features should be processed swiftly, whereas partial repetitions, in which one feature changes but others remain the same, should be processed slowly.

In a congruency task that comprises a set of only four stimuli (e.g., a typical flanker or Simon task), both CC and II trial transitions consist entirely of complete repetitions and complete alternations, whereas CI and IC trials consist entirely of partial repetitions. For example, with reference to the flanker task described above, an II trial sequence commencing with an SHS stimulus can be concluded either by an SHS stimulus (target feature repetition, distractor feature repetition, and response repetition) or by an HSH stimulus (target feature alternation, distractor feature alternation, and response alternation). In contrast, an IC trial sequence starting with an SHS stimulus can be concluded either by an SSS stimulus (target feature alternation, distractor feature repetition, and response alternation) or by an HHH stimulus (target feature repetition, distractor feature alternation, and response repetition). In Figure 1B, the hypothetical data of Figure 1A are replotted as a function of current trial congruency and type of trial transition (complete or partial, collapsed across repetitions and alternations). The graph makes it easy to appreciate that feature integration theory reconceptualizes the interaction effect of previous trial congruency $X$ current trial congruency as a main effect of congruency paired with an additive main effect of feature transition (Hommel et al., 2004).

\section{Literature Review}

Before reviewing the empirical evidence for and against the conflict adaptation and feature integration accounts, several important conceptual and methodological caveats should be noted. First, it is evident from the descrip- 
tions above that conflict adaptation and feature integration do not invoke mutually exclusive mechanisms, and it is therefore perfectly possible for both of them to affect performance simultaneously. Second, congruency tasks that comprise only two values per stimulus dimension (resulting in four possible stimuli) are not well suited for arbitrating between these accounts. In such a two-value congruency task, feature integration effects can never be fully dissociated from conflict-driven effects, since complete versus partial alternations and repetitions are perfectly confounded with types of congruency trial transitions. As an example, consider the hypothetical data depicted in Figure 1: For a two-value congruency task data set, if the data were devoid of feature integration effects (imagine there were no differences in RTs between complete vs. partial repetitions and alternations in Figure 1B), they would by definition also be devoid of congruency sequence effects, and thus of potential conflict adaptation effects (i.e., in Figure 1A, II trial RTs would not differ from CI trial RTs, and IC trial RTs would differ from CC trial RTs).

A related caveat concerns an analytical strategy that has been employed in such two-value tasks for the purpose of isolating trial sequences that are affected by feature integration effects from those that are not. This strategy involves the comparison of trial transitions that include response (or target) repetitions versus those that include response (or target) alternations (see, e.g., Nieuwenhuis et al., 2006). This strategy does not result in a clean isolation of feature integration effects, however, since feature integration should affect all partial repetitions, irrespective of whether the response remains the same and stimulus features alternate or whether the response alternates and stimulus features remain the same (Hommel et al., 2004).

An additional problem with this analytical strategy is that the comparison between response repetitions and response alternations in II stimulus sequences essentially represents a comparison between repetition priming effects on the one hand and negative priming effects on the other (Ullsperger et al., 2005), since in II response alternation trials, the distractor stimulus on the previous trial necessarily becomes the target stimulus on the current trial (Egner \& Hirsch, $2005 b$ ). Negative priming is associated with slowed responses (Neill, 1977), which thus biases this kind of analysis against finding fast responses on II response alternation trials. (It can be argued, in fact, that negative priming may reflect another type of associative feature integration effect; see Egner \& Hirsch, 2005c; Neill, 1997.) In a twovalue congruency task, the comparison of trial sequences in which responses are repeated with those in which responses alternate thus represents a complex combination of different types of feature integration and negative priming effects and does not make for unambiguous interpretation. In light of these limitations, the author maintains that only studies that employ larger stimulus sets are equipped to separate the potential contributions of conflict adaptation and feature integration processes to the congruency sequence effect, and, therefore, conclusions from such studies will be granted prominence in the ensuing review of the literature.

\section{Behavioral Data}

Congruency sequence effects in the flanker task. A number of two-value flanker studies have produced equivocal results, with some reporting that congruency sequence effects persisted after removal of complete S-R and response repetition trials (Ullsperger et al., 2005) and others reporting that these effects were eliminated (Mayr et al., 2003; Nieuwenhuis et al., 2006). A few experiments have employed more extensive stimulus sets. First, Mayr et al. (2003, Experiment 2) devised a flanker task that did not entail any stimulus or response repetitions by alternating on each trial between target and flankers that were oriented either horizontally (requiring a left or right response) or vertically (requiring an up or down response). Data gathered from this task did not display any firstorder congruency sequence effects, leading the authors to conclude that conflict-driven cognitive control processes were not a prerequisite for the congruency sequence effect. However, it has since been argued that Mayr et al.'s task may have effectively introduced a task switch between each trial (changing from vertical to horizontal stimuli and responses and back again), which may have obscured potential conflict adaptation effects (Ullsperger et al., 2005). In support of this criticism, other work has suggested that incongruent trials that precede a task switch do indeed impair performance in the new task set (Brown, Reynolds, \& Braver, 2007; Goschke, 2000).

Ullsperger et al. (2005, Experiment 2) devised a flanker paradigm with a large set of possible values (consisting of the digits 1-9) per stimulus dimension and a correspondingly large response set, which permitted them to exclude from the analysis any repetitions of stimulus components and responses from one trial to the next, thus controlling for feature integration effects. Ullsperger et al. nevertheless observed a congruency sequence effect. In another recent study, Verbruggen et al. (2006) designed a flanker task in which six possible stimulus values were mapped onto three response buttons (i.e., a six-to-three mapping with two stimulus values per response). This setup can provide control over feature integration effects, and, moreover, the six-to-three S-R mapping allowed the authors to dissociate the effects of stimulus conflict from the effects of response conflict by comparing trials that entailed stimulus conflict only (comprising an incongruent stimulus with target and flanker colors mapped onto the same response) with trials that entailed both stimulus and response conflict (comprising an incongruent stimulus with target and flanker colors mapped onto different responses) (cf. De Houwer, 2003; Eriksen \& Eriksen, 1974). When analyzing stimulus sequences devoid of stimulus and response repetitions, which thus controlled for feature integration effects, the authors nevertheless found a congruency sequence effect. Interestingly, this effect was driven by stimulus conflict rather than by response conflict.

In summary, it appears that the congruency sequence effect in the flanker task persists when first-order feature integration processes are controlled for, which has been interpreted as support for the conflict-monitoring model (Ullsperger et al., 2005; Verbruggen et al., 2006). Verbrug- 
gen et al.'s study has further constrained this interpretation by suggesting that, despite the emphasis that the conflictmonitoring model places on the role of response conflict (Botvinick et al., 2001; Botvinick et al., 2004; van Veen, Cohen, Botvinick, Stenger, \& Carter, 2001), it may be primarily the stimulus conflict component of the congruency effect that drives the adaptation process. Two caveats should be noted regarding the interpretation of these data, however. First, these conclusions are based on the assumption that any congruency sequence effect not attributable to feature integration is by default attributable to conflict adaptation, when there could, of course, be other, still unknown causes for this effect. Second, it should be noted that these results do not rule out the likely possibility that if stimulus or response features are repeated between trials, as is the case in most versions of the flanker task, feature integration effects may substantially affect the results (Mayr et al., 2003; Nieuwenhuis et al., 2006).

Congruency sequence effects in the Stroop task. The Stroop task more naturally lends itself to larger stimulus sets than do the flanker and Simon paradigms, and the majority of Stroop studies assessing congruency sequence effects could rule out feature integration effects in their conclusions (for an exception, see Egner \& Hirsch, 2005b, which employed a two-value task). Kerns et al. (2004) employed a manual response Stroop paradigm with three possible stimulus values (red, green, or blue) and analyzed only those trial transitions in which neither the word nor the ink color was repeated, thus precluding feature integration effects. Kerns et al. nevertheless observed a robust congruency sequence effect. Also employing a three-color Stroop task, Notebaert et al. (2006) found that complete alternation trials (precluding potential feature integration effects) produced a smaller congruency sequence effect than did complete and partial repetition trials (entailing potential feature integration effects), suggesting additive effects of conflict adaptation and feature integration. Furthermore, sequence effects interacted with the length of the response-to-stimulus interval (RSI): At a short RSI $(50 \mathrm{msec})$, a congruency sequence effect was evident for the complete and partial repetition trials only, whereas at a longer RSI $(200 \mathrm{msec})$, both complete alternation and complete and partial repetition trials showed congruency sequence effects. The latter findings led the authors to conclude that the presumed top-down processes underlying conflict adaptation may take longer to develop than do feature integration effects.

Egner and Hirsch (2005a) designed a face-word Stroop task with six possible stimulus values, which paired the faces of three famous actors and politicians with the names of other actors and politicians and required subjects to categorize either the face or the name dimension of the stimuli (in different versions of the task) as belonging to either an actor or a politician. Like the classic Stroop task, this design produces a semantic S-S overlap. Given this stimulus set, the authors were able to change the values of both stimulus dimensions between each trial, so that each trial transition was associated with a change in stimulus features. Furthermore, $50 \%$ of the transitions in each trial type were associated with an alternating response and $50 \%$ with a repeated response, thus equating potential feature integration effects across trial types. Nevertheless, substantial congruency sequence effects were obtained. These results have since been replicated in two very similar face-word paradigms (Egner, Etkin, Gale, \& Hirsch, in press; Etkin et al., 2006).

In summary, Stroop studies have reported that congruency sequence effects persist after first-order feature integration effects have been controlled for, which has been interpreted as documenting the effects of conflict-driven cognitive control in the Stroop paradigm (Egner et al., in press; Egner \& Hirsch, 2005a; Etkin et al., 2006; Kerns et al., 2004; Notebaert et al., 2006). Furthermore, the results of Notebaert et al. suggest that feature integration effects, if uncontrolled for, lead to substantial additional congruency sequence effects and that these effects may arise more rapidly than conflict adaptation effects do. Evidently, the conclusions above rely on the assumption that there are no causes of congruency sequence effects in the Stroop task other than conflict adaptation and feature integration effects.

Congruency sequence effects in the Simon task. Stürmer et al. (2002) conducted a series of two-value Simon task experiments, excluding S-R repetition trials, and found reliable congruency sequence effects. They interpreted these data within a conflict adaptation framework, suggesting that direct route activation (response priming by the irrelevant stimulus location) of incorrect responses was detected by a monitoring mechanism and resulted in a controlled gating of activation on the following trial, in which the influence of direct route processing on response generation was suppressed (direct route suppression). Two aspects of Stürmer et al.'s data suggested the additional presence of associative effects. First, Stürmer et al. observed substantial repetition priming effects; second, they found that under some conditions, the congruency effect was reversed after incongruent trials. A reversal of the congruency effect was replicated by Wendt et al. (2006) under conditions of very low overall Simon congruency effects. Both sets of authors noted that the reversed congruency effect could not be explained by a conflict-driven direct route suppression mechanism, which at best would have led to an elimination of congruency effects (Stürmer et al., 2002; Wendt et al., 2006). Feature integration, on the other hand, could account for a reversed congruency effect following incongruent trials in situations in which the partial repetition cost exceeded the congruency effect (cf. Wendt et al., 2006).

Another challenge to a purely conflict-driven account of the congruency sequence effect in the Simon task was presented by Hommel et al. (2004), who showed that congruency sequence effects persisted even in versions of the Simon task in which the response on a previous trial was independent of the type of stimulus encountered or in versions in which the previous trial did not require a response at all. Since these conditions presumably precluded the occurrence of response conflict on the previous trial, the authors concluded that the congruency sequence effect in the Simon task does not require conflict-driven cognitive control processes. On the other hand, Wühr (2005) designed a version of the Simon task that introduced four possible stimulus positions (the upper and lower right and left cor- 
ners of the screen) and thus could assess potential conflict adaptation effects while controlling for feature integration effects. This setup allowed the author to equate feature integration effects across trial transitions, so that each transition type was associated with equal proportions of complete alternations and partial repetitions. Congruency sequence effects were observed for both complete alternations and partial repetitions. The author concluded that congruency sequence effects in a typical Simon task likely reflect additive effects of conflict adaptation and feature integration (Wühr, 2005; see also Wühr \& Ansorge, 2005).

To summarize, different manipulations of the Simon paradigm have shown that, on the one hand, a congruency sequence effect can be observed in the likely absence of conflict adaptation effects (Hommel et al., 2004) and that, on the other hand, a congruency sequence effect can also be obtained in the likely absence of feature integration effects (Wühr, 2005). These results suggest that the congruency sequence effect in a standard (two-value) Simon task probably cannot be accounted for by either of these processes alone and might reflect their additive influences.

\section{Neurophysiological Data}

In order to enhance our understanding of how different cognitive functions are mediated by the brain, the interpretation of brain imaging data needs to be tightly constrained by well-controlled behavioral paradigms that allow for clean isolation of precisely defined cognitive operations. The congruency sequence effect holds the promise of granting such a clean window onto the operations of conflict-monitoring and cognitive control processes. However, as has been outlined above, in many instances (particularly in two-value congruency tasks), ambiguities in the interpretation of the psychological determinants of this effect necessarily introduce uncertainty into the inferences drawn from neuroimaging data. On the other hand, it can be argued that the neurophysiological data themselves can aid in the arbitration between likely explanations for the source of the congruency sequence effect. In the following, the neurophysiological predictions of the conflict adaptation and feature integration models will be examined in the light of the empirical findings in the literature.

A conflict-adaptation-based interpretation of the congruency sequence effect is particularly suited to neuroimaging studies, which tend to employ a subtractive analytical approach, because the dissection of incongruent trials into CI trials (which incur high conflict due to a low level of cognitive control) and II trials (which incur low conflict due to a high level of cognitive control) makes for a clean subtractive comparison between conflict-monitoring and cognitive control trials. By this logic, brain regions that are more active during CI trials than during II trials are probably involved in conflict-related processing, whereas brain regions more active during II than CI trials are probably involved in cognitive control processes (Botvinick et al., 1999). From a feature integration perspective, CI and II trials are subject to identical congruency effects (both being incongruent trials) but are additionally affected by different feature integration effects. II trials consist partly of repetition priming trials (complete $\mathrm{S}-\mathrm{R}$ repetitions) and partly of complete $\mathrm{S}-\mathrm{R}$ alternations, neither of which is subject to unbinding of features; both types, therefore, should require very little in the way of cognitive control effort. CI trials, on the other hand, consist entirely of partial repetitions, in which feature binding from the previous trial has to be overcome. These trials should therefore be associated with response conflict (Hommel et al., 2004) and possibly with top-down control processes that help to overcome such conflict (Mayr et al., 2003).

It follows that predictions from conflict adaptation and feature integration accounts are most clearly divergent when it comes to comparing II $>$ CI trial activity (for the CI $>$ II contrast, both models would predict activity related to response conflict to occur). In the II $>\mathrm{CI}$ trial comparison, the conflict-monitoring model predicts higher activity in regions implementing top-down biasing processes. For the flanker and Stroop tasks, these regions would include lateral prefrontal cortices (Botvinick et al., 2001), whereas for the Simon task, they would likely also feature premotor and motor cortices (Praamstra, Kleine, \& Schnitzler, 1999; Stürmer et al., 2002). The feature integration model, on the other hand, posits that II trials are associated with very little cognitive effort, as compared with CI trials, and the model would therefore make a case against a greater involvement of top-down control regions in II trials, as compared with CI trials. In line with the latter prediction, neuroimaging studies have shown that repetition priming is associated with widespread cortical deactivation in frontal and other regions (reviewed in Henson, 2003).

\section{Neurophysiological Data in the Stroop Task}

Several functional magnetic resonance imaging (fMRI) studies of the congruency sequence effect in the Stroop task have utilized the comparison between II and CI trials in order to reveal putative neural correlates of cognitive control. Egner and Hirsch (2005a) reported increased activity in the right LPFC during II trials, as compared with CI trials. LPFC activity in turn predicted increased concomitant activation in areas involved in perceptual processing of the task-relevant stimulus dimension. These results have recently been replicated in a very similar task (Egner et al., in press). In another Stroop study, Kerns et al. (2004) compared activation between fast and slow II trials, reasoning that fast II trials would reflect greater postconflict performance adjustments and thus greater levels of cognitive control. These authors found greater activation in the right LPFC for fast than for slow II trials. All of the studies above did control for feature integration effects in their design and/or analyses. In the absence of feature integration effects, the authors nevertheless observed congruency sequence effects, and, importantly, they confirmed the neurophysiological predictions of the conflict adaptation account. These data seem to supply strong evidence for the existence of this mechanism in the Stroop task. Unfortunately, comparable data are not available for the flanker task. ${ }^{3}$

\section{Neurophysiological Data in the Simon Task}

In the Simon task, a number of two-value studies have produced neurophysiological data that, in principle, are 
open to an interpretation based on feature integration effects. Kerns (2006) analyzed fMRI data from a Simon study in the same manner as did Kerns et al. (2004) (see above) and found fast II trials to be associated with higher (left) LPFC activation than slow II trials were. Note that the feature integration account would suggest that fast II trials would be subject to more priming (and fewer unbinding) processes than would slow II trials and, presumably, should therefore be associated with less LPFC activation rather than with more. Another recent study combined Stroop and Simon congruency dimensions factorially, which facilitated an interaction contrast that identified regions that were more strongly involved in putative Simon control processes than in Stroop control processes (Egner, Delano, \& Hirsch, 2007). Cognitive control in the Simon task (II > CI trials) was specifically associated with activations in the premotor and motor cortex. These fMRI studies indicated more intensive processing in frontal and premotor and motor regions in II than in CI trials (or in fast II than in slow II trials); this is consistent with predictions of the conflict adaptation account but not with predictions of the feature integration model, which holds that II trials should be associated with facilitated response selection and execution.

EEG and transcranial magnetic stimulation (TMS) studies have provided additional data of interest. First, Stürmer et al. (2002) showed that lateralized readiness potentials (LRPs) over the motor cortex (reflecting response preparation processes) that indicated incorrect response activations were smaller following incongruent trials than following congruent ones. In other words, following a congruent stimulus, incongruent stimulus position information appeared to induce an ipsilateral (incorrect) response preparation (see also Riehle, Kornblum, \& Requin, 1997; J. Zhang, Riehle, Requin, \& Kornblum, 1997), but this influence was abolished following an incongruent stimulus. These data were recently replicated and extended in Stürmer and Leuthold's (2003) study, which showed that postconflict suppression of the incorrect lateralized potential occurred at the response stage only, not earlier in the dorsal visual stream. That the control process thought to underlie the direct route suppression takes place at the stage of motor preparation is also supported by a TMS study in which the congruency sequence effect was removed when premotor processing was selectively interfered with prior to stimulus presentation (Praamstra et al., 1999). Although these results are consistent with a conflict-driven direct route suppression view, it is difficult to see how the feature integration view would explain these findings. In this model, trials that follow an incongruent stimulus are no different from trials that follow a congruent one in that they both reflect an average of equal proportions of complete and partial alternation and repetition trials (see Figure 1B) and, therefore, should not differ in terms of incorrect response preparation processes.

However, Burle et al. (2005) have recently cast doubt on a conflict-driven explanation of the Simon congruency sequence effect. In a two-value Simon task, the authors took electromyographic (EMG) recordings from the flexor pollicis brevis muscle of each hand to measure, for each trial, the degree to which the correct and incorrect response effectors were activated. They also assessed the amplitude of the electroencephalographic (EEG) errorrelated negativity (ERN) component (Falkenstein, Hohnsbein, Hoormann, \& Blanke, 1991; Gehring, Goss, Coles, Meyer, \& Donchin, 1993), which was time locked to the onset of the incorrect EMG activity. ERN amplitudes have been explicitly tied to response conflict monitoring in the ACC within the conflict-monitoring model, according to which they reflect conflict between the incorrect and the correct response activation subsequent to the execution of the incorrect response (Botvinick et al., 2001; Yeung, Cohen, \& Botvinick, 2004). Burle et al. found that correct trials that entailed incorrect EMG response activation were associated with slow responses and evoked ERN responses, suggesting the presence of response conflict. However, the degree of incorrect EMG activity (and ERN amplitudes) on a previous trial did not predict a reduced congruency effect on the next trial but, rather, was associated with slowed (but more accurate) responses and increased congruency effects. These results suggested to the authors that the congruency sequence effect in the Simon task arises independently of response conflict, at least insofar as such conflict is adequately captured by incorrect EMG activation and the ERN.

It appears difficult to reconcile these findings with the LRP data of Stürmer and colleagues (Stürmer \& Leuthold, 2003; Stürmer et al., 2002), which indicated that incorrect response activation during an incongruent trial was associated with reduced conflict (no incorrect response activation) on the subsequent trial. One aspect of Burle et al.'s (2005) results may hint at a possible explanation for this discrepancy. Burle et al. found trials that followed high levels of EMG coactivation (and ERN amplitudes) to be associated with slowed and more accurate responses. These data are reminiscent of the response pattern, termed posterror slowing, typically observed after error trials (Laming, 1968; Rabbitt, 1966). Botvinick et al. (2001) successfully modeled posterror slowing as weakened response priming, a conflict-driven general lowering of activation in response units that effectively results in a more conservative response criterion. ${ }^{4}$ Note that the conflict monitor is thought to receive its input from activation in premotor pathways prior to the peripheral EMG response activation and the eventual response execution. It is therefore possible that the trials selected by Burle et al., in which incorrect EMG activation preceded correct activation, represented trials that, at the earlier monitoring stage, displayed a higher activation for the incorrect than for the correct response. These trials may therefore subsequently have been treated as error trials by the cognitive control system (even though the correct response eventually won out), resulting in posterror slowing rather than conflict adaptation. This is, of course, a purely speculative account, and it would be very informative for future studies to acquire measures of LRP and EMG activity simultaneously, ideally in a paradigm that also controls for feature integration effects.

To summarize the neurophysiological data, studies of the Stroop and the Simon tasks have, in general, confirmed predictions derived from the conflict adaptation 
model, which expects II trials (and postincongruent trials in general) to be associated with a high level of controlled processing, and disconfirmed predictions derived from the feature integration model, which expects II trials to be associated with primed, facilitated processing (and does not expect postincongruent trials to generally differ from postcongruent ones). As a caveat, it should be noted that none of these studies set out to explicitly test predictions of the feature integration model or to directly contrast predictions from the conflict adaptation and feature integration models. Therefore, these studies have supplied only indirect, post hoc evidence in favor of the conflict adaptation account.

\section{Alternative Accounts}

One important theme that has run through this discussion of the literature is that it is currently unclear whether only conflict-driven and feature integration effects contribute to the congruency sequence effect. Thus, additional potential causes have to be identified and experimentally excluded as viable alternative explanations. As has been pointed out previously, one major concern regarding the interpretation of congruency sequence effects has revolved around the repetition of specific stimuli and responses and their mutual association (Hommel et al., 2004; Mayr et al., 2003). However, many of the studies reviewed above precluded such repetitions but, nevertheless, obtained congruency sequence effects. The present discussion, therefore, focuses on a different class of associative effects - namely, controlled processes that are driven not by conflict but, rather, by expectancy. Conflictdriven and expectancy-based effects have sometimes been treated as quasiequivalent instances of strategic processing (see, e.g., Carter et al., 2000), but in principle, they are based on rather different mechanisms, and it is possible that expectancy-based control processes contribute to the congruency sequence effect independently of conflictdriven control processes.

An expectancy-based account of the congruency sequence effect, here referred to as the repetition expectancy account, was offered in the original description of this effect by Gratton et al. (1992). This account, like the conflict-monitoring model, posits that the congruency sequence effect derives from strategic adjustments to attentional processing; however, unlike the conflict-monitoring account, it supposes that these attentional adjustments are based not on the occurrence of conflict but on subjects' expectancies regarding the nature of an upcoming trial. ${ }^{5}$ A very important and rather counterintuitive aspect of this interpretation is that these expectancies are not necessarily tied to the objective probability of a given trial type's occurring. Rather, previous work has shown that subjects who encounter a particular stimulus condition on one trial tend to expect that condition to be repeated on the next trial, even if this expectation is probabilistically quite unreasonable (Remington, 1969). Thus, the repetition expectancy account presupposes that, even in a situation in which congruent and incongruent trials are objectively equally likely to occur, subjects expect the trial following a congruent trial to be congruent and the trial following an incongruent trial to be incongruent (Gratton et al., 1992).

With respect to the flanker task, Gratton et al. (1992) reasoned that repetition expectancy would lead subjects to strategically adapt their attention to a more focused processing strategy in anticipation of an incongruent stimulus (i.e., on II and IC trials), in an attempt to minimize the detrimental influence of the flanker letters, and to a more parallel processing strategy when anticipating a congruent stimulus (i.e., on CC and CI trials), in an attempt to maximize the beneficial influence of the flanker information. It follows that on CC and II trials, subjects' predictions would be confirmed and performance would benefit from the strategic adjustments, whereas on IC and CI trials, subjects' predictions would be violated and the attentional adjustments would backfire: The beneficial effect of congruent flankers during an IC trial would be diminished, and the detrimental effects of the incongruent flankers during a CI trial would be exacerbated. The repetition expectancy account, therefore, predicts that repetition of the same congruency level would elicit faster responses than would a change of congruency levels between trials (cf. Figure 1A).

To the best knowledge of the author, no empirical study has yet ruled out the possibility that repetition expectancy effects may substantially contribute to congruency sequence effects, and this clearly constitutes an important avenue for future research in this field. The importance of this possible contribution by expectancybased effects is reinforced by the fact that they may also be invoked as an alternative explanation for another key behavioral phenomenon that has been accounted for by conflict adaptation - namely, trial type frequency effects (Botvinick et al., 2001). Many studies have shown that a high proportion of incongruent trials occurring within a block of trials is associated with reduced congruency effects, whereas a low proportion of incongruent trials is associated with enhanced congruency effects (Carter et al., 2000; Casey et al., 2000; Gratton et al., 1992; Logan \& Zbrodoff, 1979, 1982; Stürmer et al., 2002; Tzelgov, Henik, \& Berger, 1992). The conflict-monitoring model accounts for these effects by supposing that a high frequency of incongruent trials leads to a steady occurrence of conflict, resulting in maintenance of a high level of control and thus reducing the influence of distractor stimuli and the associated congruency effects. A high frequency of congruent trials results in the converse scenario: Since conflict is rare, the level of control is generally low, and thus the few incongruent trials that do occur produce high conflict and a large congruency effect (Botvinick et al., 2001).

However, it is also possible to explain trial type frequency effects on the basis of expectancy effects alone, thus entirely precluding a conflict-monitoring loop. This was Logan and Zbrodoff's $(1979,1982)$ original interpretation of these effects; they suggested that subjects form explicit probabilistic expectancies and adjust their processing strategies accordingly. Importantly, this expectancy-based view allows for the possibility that 
subjects make strategic use of information in the taskirrelevant stimulus dimension. For example, in a twocolor Stroop task with mostly incongruent stimuli, subjects may employ the task-irrelevant word meaning as a predictive shortcut to the correct response by intentionally executing the response opposite of that indicated by the word (cf. Logan \& Zbrodoff, 1979, 1982). This kind of explicit exploitation of probabilistic expectancies can account for cases in which trial type frequency produces a reversed congruency effect (Logan \& Zbrodoff, 1979, 1982; Stürmer et al., 2002). Such a reversal of the congruency effect cannot be explained by a purely conflictdriven model, in which the occurrence of conflict results in a decrease of the effect of irrelevant stimulus information. Under optimal conditions, this decrease can result in equal performance on congruent and incongruent trials but does not by itself provide the means for performing better on incongruent than on congruent trials.

This brief consideration of one alternative explanation for congruency sequence (and related) effects inspires two main conclusions. First, possible mechanisms other than feature integration and conflict adaptation clearly must be considered and their effects experimentally isolated. Second, if, as appears likely, a number of different mechanisms are found to contribute to performance, more sophisticated computational models have to be developed that can integrate the simultaneous influences of multiple strategic (and lower level) mechanisms. Promising examples of such work can already be found in the literature. For instance, Jones, Cho, Nystrom, Cohen, and Braver (2002) have integrated expectancy-based mechanisms (cf. Cho et al., 2002) with a conflict monitor ${ }^{6}$ to account for behavioral and ACC activity findings in the context of (nonconflict) two-alternative forced choice tasks. More recently, Brown et al. (2007) have combined the workings of a conflict-driven control mechanism that facilitates task-relevant processing with a task/response switch detector that induces a shift toward a more conservative speed-accuracy trade-off. This model successfully captured interactions among congruency sequence and taskswitching effects found in empirical data.

\section{Conclusions and Outlook}

On the basis of the survey of the congruency sequence effect literature, a number of general conclusions may be drawn. First, the behavioral data show that neither conflict adaptation nor feature integration effects can account for all instances of the congruency sequence effect (Burle et al., 2005; Hommel et al., 2004; Mayr et al., 2003; Ullsperger et al., 2005; Verbruggen et al., 2006; Wühr, 2005), and these data suggest that the sequence effect in a typical two-value congruency task likely entails additive contributions of conflict adaptation and feature integration effects (Notebaert et al., 2006; Stürmer et al., 2002; Wühr \& Ansorge, 2005). The main research endeavor of interest in this area, therefore, may lie not in the dichotomous question of whether congruency sequence effects are accounted for entirely by one or the other of these mechanisms but, rather, with how to best isolate their respective contribu- tions. The author has argued here that this isolation could best be achieved through the use of a stimulus and response set large enough to allow complete and partial feature alternations to be equated across different trial types.

Second, studies that did control for feature integration effects through the use of large stimulus and response sets have produced important findings concerning the mechanisms that may underlie conflict-driven cognitive control. Of particular interest is the finding that conflict adaptation in S-S overlap tasks (such as the flanker and Stroop tasks) may be driven by stimulus conflict rather than by response conflict (Verbruggen et al., 2006). This result is at odds with the emphasis of the conflict-monitoring model on conflict at the response selection level (Botvinick et al., 2001; Botvinick et al., 2004; van Veen et al., 2001). Although the possibility that conflict at other processing levels drives control (and ACC activity) has been explicitly acknowledged (Botvinick et al., 2001), the computational architecture of the conflict-monitoring model is currently not equipped to distinguish between stimulus conflict and response conflict components of the congruency effect in S-S overlap tasks, since the architecture does not entail an intermediate layer in which different stimulus streams converge and compete for representation (cf. H. Zhang et al., 1999). The behavioral findings of Verbruggen et al. (2006), in combination with recent fMRI data showing that the ACC is activated by stimulus conflict (van Veen \& Carter, 2005), may motivate further refinement of this model.

Third, data from neurophysiological studies have largely supported the predictions of the conflict adaptation account. In addition, these data provide further clues to a more fine-grained understanding of conflict-driven control mechanisms. Foremost, a strong case can be made for the claim that conflict resolution in $\mathrm{S}-\mathrm{S}$ overlap tasks (notably the Stroop task) is mediated by a different process (and associated neural circuitry) than is conflict resolution in irrelevant S-R overlap tasks - that is, the Simon task (Egner, Delano, \& Hirsch, 2007). Specifically, conflict resolution in the Stroop task likely involves the excitatory biasing of task-relevant stimulus processing (Cohen et al., 1990; Egner, Delano, \& Hirsch, 2007; Egner et al., in press; Egner \& Hirsch, 2005a), whereas conflict resolution in the Simon task likely involves the inhibition of direct route response-priming processes (Egner, Delano, \& Hirsch, 2007; Praamstra et al., 1999; Stürmer \& Leuthold, 2003; Stürmer et al., 2002). In keeping with these assumptions, two recent studies have shown that the congruency sequence effect is conflict specific, in that Stroop conflict on a previous trial improves the resolution of Stroop (S-S overlap) conflict on the current trial but does not affect the resolution of Simon (irrelevant S-R overlap) conflict and vice versa (Egner, Delano, \& Hirsch, 2007; Wendt et al., 2006). These results converge to suggest that different types of conflict are resolved via distinct conflict control loops, implying that conflict-driven cognitive control processes should not be thought of as emanating from a single, central resource but, rather, from a collection of conflict-specific regulatory loops that may operate 
in parallel. An important question is whether the proposed monitoring function of the $\mathrm{ACC} /$ medial prefrontal cortex is preserved across different types of conflict.

Finally, even when controlling for feature integration effects, the interpretation of congruency sequence effects as reflecting conflict-driven control processes still hinges on the assumption that no other processes additionally impact this effect. This assumption may well be incorrect, and it is therefore crucial to identify possible additional contributors to this effect, such as expectancy-based control processes. One exciting future challenge in improving our understanding of cognitive control processes then lies with modeling the integration of conflict-driven performance adjustments with other cognitive control mechanisms, as well as with lower level mechanisms that determine behavior (see, e.g., Brown et al., 2007; Jones et al., 2002).

\section{AUTHOR NOTE}

Correspondence concerning this article should be addressed to T. Egner, Northwestern University, Feinberg School of Medicine, 320 East Superior Street, Searle 11, Chicago, IL 60611 (e-mail: t-egner@northwestern.edu).

\section{REFERENCES}

Botvinick, M. M., Braver, T. S., Barch, D. M., Carter, C. S., \& Cohen, J. D. (2001). Conflict monitoring and cognitive control. Psychological Review, 108, 624-652.

Botvinick, M. M., Cohen, J. D., \& Carter, C. S. (2004). Conflict monitoring and anterior cingulate cortex: An update. Trends in Cognitive Sciences, 8, 539-546.

Botvinick, M. M., Nystrom, L. E., Fissell, K., Carter, C. S., \& Cohen, J. D. (1999). Conflict monitoring versus selection-for-action in anterior cingulate cortex. Nature, 402, 179-181.

Brown, J. W., Reynolds, J. R., \& Braver, T. S. (2007). A computational model of fractionated conflict-control mechanisms in taskswitching. Cognitive Psychology, 55, 37-85.

Burle, B., Allain, S., Vidal, F., \& Hasbroucq, T. (2005). Sequential compatibility effects and cognitive control: Does conflict really matter? Journal of Experimental Psychology: Human Perception \& Performance, 31, 831-837.

Carter, C. S., Macdonald, A. M., Botvinick, M. [M.], Ross, L. L., Stenger, V. A., Noll, D., \& Cohen, J. D. (2000). Parsing executive processes: Strategic vs. evaluative functions of the anterior cingulate cortex. Proceedings of the National Academy of Sciences, 97, 1944-1948.

Casey, B. J., Thomas, K. M., Welsh, T. F., Badgaiyan, R. D., Eccard, C. H., Jennings, J. R., \& Crone, E. A. (2000). Dissociation of response conflict, attentional selection, and expectancy with functional magnetic resonance imaging. Proceedings of the National Academy of Sciences, 97, 8728-8733.

Cho, R. Y., Nystrom, L. E., Brown, E. T., Jones, A. D., Braver, T. S., Holmes, P. J., \& Cohen, J. D. (2002). Mechanisms underlying dependencies of performance on stimulus history in a two-alternative forced-choice task. Cognitive, Affective, \& Behavioral Neuroscience, 2, 283-299.

Cohen, J. D., Botvinick, M. [M.], \& Carter, C. S. (2000). Anterior cingulate and prefrontal cortex: Who's in control? Nature Neuroscience, 3, 421-423.

Cohen, J. D., Dunbar, K., \& McClelland, J. L. (1990). On the control of automatic processes: A parallel distributed processing account of the Stroop effect. Psychological Review, 97, 332-361.

Cohen, J. D., Servan-Schreiber, D., \& McClelland, J. L. (1992). A parallel distributed processing approach to automaticity. American Journal of Psychology, 105, 239-269.

De Houwer, J. (2003). On the role of stimulus-response and stimulusstimulus compatibility in the Stroop effect. Memory \& Cognition, 31, 353-359.

Durston, S., Davidson, M. C., Thomas, K. M., Worden, M. S., Tottenham, N., Martinez, A., ET AL. (2003). Parametric manipulation of conflict and response competition using rapid mixed-trial eventrelated fMRI. NeuroImage, 20, 2135-2141.

Egner, T., Delano, M., \& Hirsch, J. (2007). Separate conflict-specific cognitive control mechanisms in the human brain. NeuroImage, $\mathbf{3 5}$, 940-948.

Egner, T., Etkin, A., Gale, S., \& Hirsch, J. (in press). Dissociable neural systems resolve conflict from emotional versus nonemotional distractors. Cerebral Cortex.

EgNeR, T., \& HiRsCH, J. (2005a). Cognitive control mechanisms resolve conflict through cortical amplification of task-relevant information. Nature Neuroscience, 8, 1784-1790.

EgNeR, T., \& HiRSCH, J. (2005b). The neural correlates and functional integration of cognitive control in a Stroop task. NeuroImage, 24, 539-547.

EGNER, T., \& HiRSCH, J. (2005c). Where memory meets attention: Neural substrates of negative priming. Journal of Cognitive Neuroscience, 17, 1774-1784.

ERIKSEN, B. A., \& ERIKSEN, C. W. (1974). Effects of noise letters upon the identification of a target letter in a nonsearch task. Perception \& Psychophysics, 16, 143-149.

Etkin, A., Egner, T., Peraza, D. M., Kandel, E. R., \& Hirsch, J. (2006). Resolving emotional conflict: A role for the rostral anterior cingulate cortex in modulating activity in the amygdala. Neuron, 51, 871-882.

Falkenstein, M., Hohnsbein, J., Hoormann, J., \& Blanke, L. (1991). Effects of crossmodal divided attention on late ERP components: II. Error processing in choice reaction tasks. Electroencephalography \& Clinical Neurophysiology, 78, 447-455.

Gehring, W. J., Goss, B., Coles, M. G. H., Meyer, D. E., \& DonCHIN, E. (1993). A neural system for error detection and compensation. Psychological Science, 4, 385-390.

GoschKe, T. (2000). Intentional reconfiguration and involuntary persistence in task-set switching. In S. Monsell \& J. Driver (Eds.), Control of cognitive processes: Attention and performance XVIII (pp. 331-355). Cambridge, MA: MIT Press.

Gratton, G., Coles, M. G. H., \& Donchin, E. (1992). Optimizing the use of information: Strategic control of activation of responses. Journal of Experimental Psychology: General, 121, 480-506.

Henson, R. N. (2003). Neuroimaging studies of priming. Progress in Neurobiology, 70, 53-81.

Hommel, B., Proctor, R. W., \& Vu, K.-P. L. (2004). A feature-integration account of sequential effects in the Simon task. Psychological Research, 68, $1-17$

Jones, A. D., Cho, R. Y., Nystrom, L. E., Cohen, J. D., \& Braver, T. S. (2002). A computational model of anterior cingulate function in speeded response tasks: Effects of frequency, sequence, and conflict. Cognitive, Affective, \& Behavioral Neuroscience, 2, 300-317.

KERNS, J. G. (2006). Anterior cingulate and prefrontal cortex activity in an fMRI study of trial-to-trial adjustments on the Simon task. NeuroImage, 33, 399-405.

Kerns, J. G., Cohen, J. D., MacDonald, A. W., III, Cho, R. Y., Stenger, V. A., \& CARTer, C. S. (2004). Anterior cingulate conflict monitoring and adjustments in control. Science, 303, 1023-1026.

Kornblum, S., HasbroucQ, T., \& Osman, A. (1990). Dimensional overlap: Cognitive basis for stimulus-response compatibility-a model and taxonomy. Psychological Review, 97, 253-270.

Kornblum, S., \& LeE, J. W. (1995). Stimulus-response compatibility with relevant and irrelevant stimulus dimensions that do and do not overlap with the response. Journal of Experimental Psychology: Human Perception \& Performance, 21, 855-875.

LAMING, D. (1968). Information theory of choice reaction times. London: Academic Press.

Logan, G. D., \& ZBrodoff, N. J. (1979). When it helps to be misled: Facilitative effects of increasing the frequency of conflicting stimuli in a Stroop-like task. Memory \& Cognition, 7, 166-174.

Logan, G. D., \& ZBRodoff, N. J. (1982). Constraints on strategy construction in a speeded discrimination task. Journal of Experimental Psychology: Human Perception \& Performance, 8, 502-520.

Lu, C.-H., \& Proctor, R. W. (1995). The influence of irrelevant location information on performance: A review of the Simon and spatial Stroop effects. Psychonomic Bulletin \& Review, 2, 174-207.

MacLeod, C. M. (1991). Half a century of research on the Stroop effect: An integrative review. Psychological Bulletin, 109, 163-203. 
Mayr, U., Awh, E., \& Laurey, P. (2003). Conflict adaptation effects in the absence of executive control. Nature Neuroscience, $\mathbf{6}, 450-452$.

Milham, M. P., Banich, M. T., Webb, A., Barad, V., Cohen, N. J., Wszalek, T., \& Kramer, A. F. (2001). The relative involvement of anterior cingulate and prefrontal cortex in attentional control depends on nature of conflict. Cognitive Brain Research, 12, 467-473.

MilleR, E. K., \& Cohen, J. D. (2001). An integrative theory of prefrontal cortex function. Annual Review of Neuroscience, 24, 167-202.

NeILL, W. T. (1977). Inhibitory and facilitatory processes in selective attention. Journal of Experimental Psychology: Human Perception \& Performance, 3, 444-450.

NeILL, W. T. (1997). Episodic retrieval in negative priming and repetition priming. Journal of Experimental Psychology: Learning, Memory, \& Cognition, 23, 1291-1305.

Nieuwenhuis, S., Stins, J. F., Posthuma, D., Polderman, T. J. C., Boomsma, D. I., \& DE Geus, E. J. (2006). Accounting for sequential trial effects in the flanker task: Conflict adaptation or associative priming? Memory \& Cognition, 34, 1260-1272.

Notebaert, W., Gevers, W., Verbruggen, F., \& Liefooghe, B. (2006). Top-down and bottom-up sequential modulations of congruency effects. Psychonomic Bulletin \& Review, 13, 112-117.

Pashler, H., \& BaYlis, G. C. (1991). Procedural learning: II. Intertrial repetition effects in speeded choice tasks. Journal of Experimental Psychology: Learning, Memory, \& Cognition, 17, 33-48.

Posner, M. I., \& DiGirolamo, G. J. (1998). Executive attention: Conflict, target detection, and cognitive control. In R. Parasuraman (Ed.), The attentive brain (pp. 401-423). Cambridge, MA: MIT Press.

Praamstra, P., Kleine, B. U., \& Schnitzler, A. (1999). Magnetic stimulation of the dorsal premotor cortex modulates the Simon effect. NeuroReport, 10, 3671-3674.

RaвbitT, P. M. A. (1966). Errors and error correction in choice-response tasks. Journal of Experimental Psychology, 71, 264-272.

REMINGTON, R. J. (1969). Analysis of sequential effects in choice reaction times. Journal of Experimental Psychology, 82, 250-257.

Ridderinkhof, K. R., Ullsperger, M., Crone, E. A., \& NieuwenHUIS, S. (2004). The role of the medial frontal cortex in cognitive control. Science, 306, 443-447.

Riehle, A., Kornblum, S., \& Requin, J. (1997). Neuronal correlates of sensorimotor association in stimulus-response compatibility. Journal of Experimental Psychology: Human Perception \& Performance, 23, $1708-1726$.

SimON, J. R. (1969). Reactions toward the source of stimulation. Journal of Experimental Psychology, 81, 174-176.

StROOP, J. R. (1935). Studies of interference in serial verbal reactions. Journal of Experimental Psychology, 18, 643-662.

Stürmer, B., \& Leuthold, H. (2003). Control over response priming in visuomotor processing: A lateralized event-related potential study. Experimental Brain Research, 153, 35-44.

Stürmer, B., Leuthold, H., Soetens, E., Schröter, H., \& SomMER, W. (2002). Control over location-based response activation in the Simon task: Behavioral and electrophysiological evidence. Journal of Experimental Psychology: Human Perception \& Performance, 28 $1345-1363$

Tzelgov, J., HeniK, A., \& Berger, J. (1992). Controlling Stroop effects by manipulating expectations for color words. Memory \& Cognition, 20, 727-735.

Ullsperger, M., Bylsma, L. M., \& Botvinick, M. M. (2005). The conflict adaptation effect: It's not just priming. Cognitive, Affective, \& Behavioral Neuroscience, 5, 467-471.

van Veen, V., \& Carter, C. S. (2005). Separating semantic conflict and response conflict in the Stroop task: A functional MRI study. NeuroImage, 27, 497-504.

van Veen, V., Cohen, J. D, Botvinick, M. M., Stenger, V. A., \& CARTER, C. S. (2001). Anterior cingulate cortex, conflict monitoring, and levels of processing. Neurolmage, 14, 1302-1308.

Verbruggen, F., Notebaert, W., Liefooghe, B., \& Vandierendonck, A.
(2006). Stimulus- and response-conflict-induced cognitive control in the flanker task. Psychonomic Bulletin \& Review, 13, 328-333.

Wendt, M., Kluwe, R. H., \& Peters, A. (2006). Sequential modulations of interference evoked by processing task-irrelevant stimulus features. Journal of Experimental Psychology: Human Perception \& Performance, 32, 644-667.

WüHR, P. (2005). Evidence for gating of direct response activation in the Simon task. Psychonomic Bulletin \& Review, 12, 282-288.

WÜHR, P., \& ANSORGE, U. (2005). Exploring trial-by-trial modulations of the Simon effect. Quarterly Journal of Experimental Psychology, 58A, 705-731.

Yeung, N., Cohen, J. D., \& Botvinick, M. M. (2004). The neural basis of error detection: Conflict monitoring and the error-related negativity. Psychological Review, 111, 931-959.

Zhang, H., \& Kornblum, S. (1998). The effects of stimulus-response mapping and irrelevant stimulus-response and stimulus-stimulus overlap in four-choice Stroop tasks with single-carrier stimuli. Journal of Experimental Psychology: Human Perception \& Performance, 24, 3-19.

Zhang, H., Zhang, J., \& Kornblum, S. (1999). A parallel distributed processing model of stimulus-stimulus and stimulus-response compatibility. Cognitive Psychology, 38, 386-432.

Zhang, J., Riehle, A., Requin, J., \& Kornblum, S. (1997). Dynamics of single neuron activity in monkey primary motor cortex related to sensorimotor transformation. Journal of Neuroscience, 17, 2227-2246.

\section{NOTES}

1. This is not the case in versions of the flanker task that employ left- and right-pointing arrows as relevant and irrelevant stimuli, since arrows are intrinsically associated (and thus overlap) with a particular response direction/effector. An arrow flanker task can therefore be argued to entail both relevant and irrelevant $\mathrm{S}-\mathrm{R}$ overlaps, in addition to an $\mathrm{S}-\mathrm{S}$ overlap.

2. In the more traditional version of the Stroop task, subjects are required to vocally name the ink color of the word stimulus. This response dimension overlaps with both the relevant and the irrelevant stimulus dimensions and thus results in relevant and irrelevant S-R overlaps, both of which can be shown to produce substantial congruency effects (H. Zhang \& Kornblum, 1998).

3. One flanker study focused exclusively on the comparison of CI $>$ II trial activation (Botvinick et al., 1999), whereas others concentrated on analyzing higher order sequences (Durston et al., 2003) or probabilistic expectancy effects (Casey et al., 2000).

4. Within the conflict-monitoring model, the consequences of conflict on error trials (a lowering of general response activation and slow, accurate responses on the subsequent trial) are quite different from the consequences of conflict on correct trials (an increase in attentional focus and fast, accurate responses on the subsequent trial). How these two distinct types of performance adjustments following conflict may operate simultaneously has been speculated upon (Cohen et al., 2000) but not yet tested, since Botvinick et al. (2001) did not implement posterror slowing and conflict adaptation within the same model.

5. Although the term expectancy is colloquially associated with awareness, the expectancy of a particular trial type could, in principle, be implicit rather than consciously accessible (i.e., explicit).

6. Note that Jones et al. (2002) modeled the consequence of conflict as a reduction in response priming that is similar to the posterror slowing mechanism modeled in Botvinick et al. (2001) but dissimilar to the increased strategic biasing toward processing of task-relevant stimulus features that is thought to mediate the conflict adaptation effect (Botvinick et al., 2001).

(Manuscript received February 6, 2007; revision accepted for publication July 16, 2007.) 